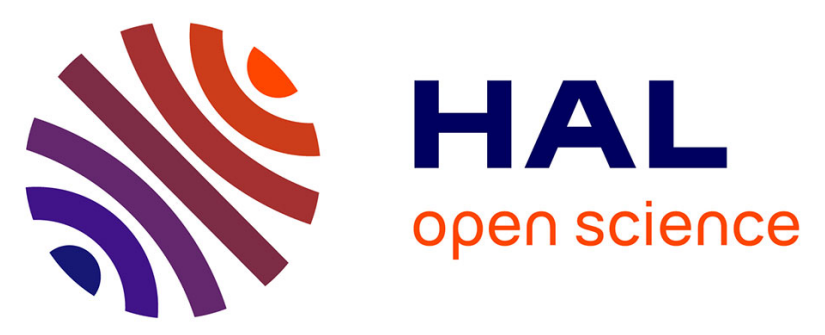

\title{
Combinatorial expression of microtubule-associated EB1 and ATIP3 biomarkers improves breast cancer prognosis
}

Sylvie Rodrigues-Ferreira, Anne Nehlig, Clarisse Monchecourt, Sarah Nasr, Laetitia Fuhrmann, Magali Lacroix-Triki, Ingrid Garberis, Véronique Scott, Suzette Delaloge, Barbara Pistilli, et al.

\section{To cite this version:}

Sylvie Rodrigues-Ferreira, Anne Nehlig, Clarisse Monchecourt, Sarah Nasr, Laetitia Fuhrmann, et al.. Combinatorial expression of microtubule-associated EB1 and ATIP3 biomarkers improves breast cancer prognosis. Breast Cancer Research and Treatment, 2019, 173 (3), pp.573-583. 10.1007/s10549018-5026-1 . hal-02411558

\section{HAL Id: hal-02411558 \\ https://hal.science/hal-02411558}

Submitted on 26 Aug 2021

HAL is a multi-disciplinary open access archive for the deposit and dissemination of scientific research documents, whether they are published or not. The documents may come from teaching and research institutions in France or abroad, or from public or private research centers.
L'archive ouverte pluridisciplinaire HAL, est destinée au dépôt et à la diffusion de documents scientifiques de niveau recherche, publiés ou non, émanant des établissements d'enseignement et de recherche français ou étrangers, des laboratoires publics ou privés. 
Combinatorial expression of microtubule-associated EB1 and ATIP3 biomarkers improves breast cancer prognosis

Sylvie RODRIGUES-FERREIRA ${ }^{1,2,3,4}$, Anne NEHLIG ${ }^{1,2,3}$, Clarisse MONCHECOURT ${ }^{1,2,3}$, Sarah NASR $^{5}$, Laetitia FUHRMANN ${ }^{6}$, Magali LACROIXTRIKI $^{5}$, Ingrid GARBERIS ${ }^{1,2,3}$, Véronique SCOTT ${ }^{1,2,3}$, Suzette DELALOGE ${ }^{5}$, Barbara PISTILLI $^{5}$, Philippe VIELH ${ }^{7}$, Thierry DUBOIS ${ }^{8}$, Anne VINCENT-SALOMON ${ }^{6}$, Fabrice ANDRE ${ }^{1,2,3}$, Clara NAHMIAS ${ }^{1,2,3 \#}$

1. INSERM U981, Department of Molecular Medicine, Gustave Roussy Cancer Center, 114 rue Edouard Vaillant, 94800 Villejuif, France.

2. LabEx LERMIT, University Paris Saclay, 5 Rue J-B Clément, 92296 Châtenay-Malabry, France.

3. University Paris Sud, 63 rue Gabriel Peri, 94270 Le Kremlin-Bicetre, France.

4. Inovarion SAS, 75013 Paris, France.

5. Department of Medical Oncology, Gustave Roussy Cancer Center, 114 rue Edouard Vaillant, 94800 Villejuif, France.

6. Institut Curie, PSL Research University, Pathology Department, 26 rue d'Ulm, F-75005, Paris, France.

7. Department of Medical Biology and Pathology, Gustave Roussy, Cancer Center 114 rue Edouard Vaillant, 94805 Villejuif, France.

8. Institut Curie, PSL Research University, Translational Research Department, Breast Cancer Biology Group, Paris, France.

\# to whom correspondence should be sent : Gustave Roussy Cancer Center, Dept of Molecular Medicine, 114 rue Edouard Vaillant, 94800 Villejuif, France.

Email : clara.nahmias@inserm.fr

Keywords

MAPRE1, MTUS1, diagnosis, prognosis, breast cancer, biomarkers combination 


\begin{abstract}
Purpose. The identification of molecular biomarkers for classification of breast cancer is needed to better stratify the patients and guide therapeutic decisions. The aim of this study was to investigate the value of MAPRE1 gene encoding microtubule-end binding proteins EB1 as a biomarker in breast cancer and evaluate whether combinatorial expression of MAPRE1 and MTUS1 gene encoding EB1-negative regulator ATIP3 may improve breast cancer diagnosis and prognosis.
\end{abstract}

Methods. Probeset intensities for MAPRE1 and MTUS1 genes were retrieved from Exonhit splice array analyses of 45 benign and 120 malignant breast tumors for diagnostic purposes. $\underline{\text { Transcriptomic analyses (U133 Affymetrix array) of one exploratory cohort of } 150 \text { invasive }}$ breast cancer patients and two independent series of 130 and 155 samples were compared with clinical data of the patients for prognostic studies. A tissue microarray from an independent cohort of 212 invasive breast tumors was immunostained with anti-EB1 and anti-ATIP3 antibodies.

Results. We show that MAPRE1 gene is a diagnostic and prognostic biomarker in breast cancer. High MAPRE1 levels correlate with tumor malignancy, high histological grade and poor clinical outcome. Combination of high-MAPRE1 and low-MTUS1 levels in tumors is significantly associated with tumor agressiveness and reduced patient survival. IHC studies of combined EB1/ATIP3 protein expression confirmed these results.

Conclusions. These studies emphasize the importance of studying combinatorial expression of EB1 and ATIP3 genes and proteins rather than each biomarker alone. A population of highly aggressive breast tumors expressing high-EB1/low-ATIP3 may be considered for the development of new molecular therapies. 


\section{Introduction}

Breast cancer is a complex disease whose clinical management relies on well-established clinico-pathological characteristics and molecular biomarkers. The emergence of highthroughput techniques for molecular profiling of breast tumors, such as DNA arrays and RNAseq, has allowed extensive progress in the diagnosis, classification and prognosis of breast tumors. The availability of large molecular databases now provides the opportunity to rapidly analyze individual and combinatorial expression of genes in cohorts of breast cancer patients, which may accelerate the identification of novel molecular biomarkers that are urgently needed for better stratifying breast cancer patients and deciding the type of therapy to be administered. The microtubule (MT) cytoskeleton plays a key role in various biological processes such as intracellular transport, cell migration and mitosis, all of which are altered in cancer. MTs are polarized protofilaments that constantly alternate between phases of polymerization (growth) and depolymerization (shrinkage) at their plus ends [1,2]. This dynamic behavior is essential for MTs to explore the cytosol and ensure cell homeostasis, and is tightly regulated by a wide number of MT-associated proteins (MAPs) [3]. Any defect in these regulatory proteins may alter the organisation and/or function of MTs, with major consequences on cancer initiation or progression.

End-binding protein 1 (EB1) is the leader member of a subfamily of MAPs including EB2 and EB3, encoded by homologous genes designated MAPRE1, MAPRE2 and MAPRE3, respectively [4]. EB1 and EB3 have been extensively studied, EB1 being ubiquitous and EB3 being predominant in the brain, whereas EB2 remains less characterized [5]. EB1 preferentially binds and accumulates at growing MT ends and is considered a surrogate marker of MT dynamics [6]. Upon binding to MT ends, EB1 accelerates the rate of catastrophe (rapid depolymerization) and thus also functionnally contributes to MT-end maturation and dynamic 
instability [7]. In addition, EB1 recruits a large number of regulatory proteins at MT plus ends, The pivotal role of EB1 on MT dynamics suggests that alterations of EB1 expression or function may have important consequences in cancer. Indeed, up-regulation of EB1 protein in tumor samples has been reported in breast cancer [11], glioblastoma [12], hepatocarcinoma [13], oral [14] and colorectal cancer $[15,16]$. In breast cancer, high EB1 protein levels have been shown to correlate with tumor malignancy and high tumor grade [11]. However, the status of MAPRE1 gene encoding EB1, and its paralogs MAPRE2 and MAPRE3, has not yet been evaluated in breast cancer, and it remains to be established whether EB1 represents a prognostic biomarker of breast cancer patient survival.

Recent studies have identified several structural MAPs as endogenous antagonists of EB1 functions at MT plus ends [5]. Among them, the ATIP3 protein encoded by candidate tumor suppressor gene MTUS1 has been described as a prognostic biomarker down-regulated in invasive breast cancer $[17,18]$. ATIP3 is a potent MT-stabilizer that markedly reduces breast tumor growth and distant metastasis [18]. ATIP3 directly binds to EB1 in the cytosol and prevents its turnover and accumulation at growing MT ends, thereby reducing MT dynamics and cell polarity [19]. These data suggest that altered expression of either ATIP3 or EB1 in breast tumors may impact the levels of intracellular ATIP3-EB1 molecular complexes that govern EB1 function at MT plus ends. This raises the interesting possibility that deleterious effects of high EB1 levels in breast tumors may be moderated by high levels of ATIP3 whereas tumors with high-EB1 and low-ATIP3 levels may remain more aggressive.

The present study investigates the value of MAPRE genes, alone or in combination with MTUS1, for molecular classification, diagnosis and prognosis of breast cancer patients. We show that high MAPRE1 - but not MAPRE2 and MAPRE3 - levels correlate with tumor malignancy and poor clinical outcome for breast cancer patients, and that combinatorial 
analysis of MAPRE1 and MTUS1 expression refines breast cancer diagnosis and prognosis compared to MAPRE1 alone. 


\section{Methods}

\section{Breast tumor samples and gene arrays}

In a first cohort of patients (Cohort\#1) designed for tumor classification, samples were obtained by fine-needle aspiration of breast lesions from patients referred to the breast diagnosis center of the Gustave Roussy Center (Villejuif, France) between 2006 and 2007 [20]. 165 samples, among which 45 benign and 120 malignant tumors, were profiled on a Splice Array (Exonhit, France) [21]. Patient characteristics and probeset intensities are presented in Supplemental Table S1.

The exploratory cohort of breast cancer patients (Cohort\#2), designed for prognostic purposes, comprises 150 infiltrating breast carcinomas obtained from patients who were included between 1988 and 1999 in the prospective database initiated in 1981 by the Institut Curie Breast cancer group (Curie, Paris, France) [17]. Samples were analyzed by Affymetrix HG-U133 DNA array hybridization as described $[17,18,22]$. Patients included in the study were aged 33 to 88 and were treated by radiotherapy combined or not with hormono- or chemotherapy after chirurgical resection of the tumor. Immunohistological levels of estrogen receptor (ER) and progesterone receptor (PR) were recorded according to standardized guidelines using $10 \%$ as the cut-off for $\underline{\text { ER and PR positive cells [23]. For human epidermal growth factor receptor-2 (HER2), only }}$ membrane staining was considered with a $30 \%$ cut-off as recommended [24]. Patient characteristics and probeset intensities are presented in Supplemental Table S2. Two independent series of breast cancer patients, designated Cohort\#3 [25, 26] and Cohort\#4 [18] are described in Supplemental Methods and Supplemental Tables S3 and S4.

\section{Immunohistochemical analysis of breast cancer tissue microarray (TMA)}

Samples of invasive ductal carcinomas (IDCs) were surgically removed before any radiation, hormonal or chemotherapy treatment at Institut Curie from 2005 to 2006 [27]. Patient 
characteristics and clinical data are presented in Supplemental Table S5. TMA consisted of replicate 1-mm diameter tumor cores selected from whole-tumor tissue section in two most representative tumor areas (high tumor cell density) of each tumor sample. Alcohol formalin acetic acid-fixed paraffin-embedded samples were analyzed by immunohistochemistry (IHC) staining using EnVision FLEX kit (Dako) according to the manufacturer's instructions. For EB1 staining, heat-mediated antigen retrieval was performed in Ethylene-Diamine-TetraAcetic acid (EDTA) buffer $\mathrm{pH} 6$ in water bath for $30 \mathrm{~min}$ and monoclonal mouse anti-EB1 antibodies (BD Biosciences) diluted 1:300 were incubated overnight at $4^{\circ} \mathrm{C}$. ATIP3 staining was performed as previously described [17] using monoclonal anti-MTUS1 antibodies (Abnova) diluted $1: 100$. Slides were counterstained with hematoxylin. HeLa cells transfected with control siRNA were used as positive control for EB1 staining, and cells transfected for 48h with EB1-specific siRNA (Dharmacon) [19] were considered as negative control. Silencing efficiency was validated by immunoblotting using anti-EB1 antibodies (BD Biosciences) diluted $1: 1000$ and anti-alpha-tubulin antibodies (Sigma) diluted $1: 1000$ as internal control. EB1 immunoreactivity in tissue sections was classified semi-quantitatively into 5 classes $(0$, $\leq 1,1,2,3)$ according to the intensity of cytoplasmic staining of tumor cells. Scores for EB1 $\underline{\text { immunostaining were defined as } 0 \text { (undetectable staining on high-power field (x40), <1 (only }}$ visible on high-power field (x40), 1 (detected on medium-power field (x10-x20) but well visualized on high-power field (x40), 2 (detected on low-power field (x4-x5) but well visualized on medium-power field (x20), and 3 (well visualized on low-power field (x4-x5). Samples were independently evaluated by two pathologists of different institutes. EB1 expression was classified on a semi-quantitative basis, immunostaining was considered weak for a score from

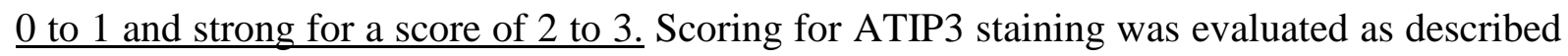
[17]. 


\section{Statistical analysis}

Statistical analyses were done using JMP-7 and GraphPad Prism 6.0 softwares. The association between clinicopathological characteristics and the expression level of either MAPRE1, MTUS1, or their combination were calculated using the Mann-Whitney test for continuous variables and using the chi-squared and the Fisher exact tests for dichotomized variables. Dot plot analyses were done using Mann-Whitney test. Overall survival (OS) and Relapse-free survival (RFS) curves were plotted according to the method of Kaplan-Meier and compared by the log-rank test. $P<0.05$ was considered statistically significant. 


\section{Results}

\section{Combinatorial expression of MAPRE1 and MTUS1 genes improves breast cancer}

diagnosis

Expression levels of the MAPRE1 gene encoding End-Binding protein EB1 were evaluated in a splice-array profiling of 165 breast samples including 120 malignant cancers and 45 benign lesions (Cohort\#1) [20]. Only $\mathrm{T}$ probesets - that identify expressed exons - were taken into account to avoid potential bias due to alternative exon splicing. Analysis of probesets intensities revealed that MAPRE1 transcripts are significantly higher in malignant compared to benign breast tumors (Fig.1a and Supplemental Fig.S1). Heatmap analysis of MAPRE1 gene expression was then used to classify tumor samples according to high or low levels of EB1 transcripts (Fig.1b). As shown in Fig. 1c and Table 1, 65\% of low-MAPRE1 expressing tumors were malignant compared to $79,6 \%$ of those expressing high-MAPRE1 levels, further indicating that elevated expression of MAPRE1 gene is associated with tumor malignancy. In contrast, expression levels of paralog MAPRE2 and MAPRE3 genes encoding EB2 and EB3 proteins, respectively, were either decreased (Supplemental Fig.S2a) or unchanged (Supplemental Fig.S2b) in cancer samples compared to benign lesions.

Expression of the MTUS1 gene, whose major product ATIP3 antagonizes EB1 functions [5, 19], was analyzed in the same samples (Supplemental Table S1). Analysis of MTUS1 probesets intensities (Fig.1d and Supplemental Fig.S3) revealed that MTUS1 levels are significantly decreased in malignant breast tumors compared to benign lesions. Classification of samples into clusters expressing high- and low-MTUS1 using heatmap analysis (Fig.1e) further indicated that $93,6 \%$ of low-MTUS1 tumors were malignant compared to $54 \%$ of tumors with high-MTUS1 levels (Fig.1f, Table 1).

We next evaluated the diagnostic value of combining MAPRE1 and MTUS1 gene expression. Tumors expressing high-MAPRE1 and low-MTUS1 levels included a majority (97.7\%) of 
malignant samples, compared to $45.2 \%$ among those expressing low-MAPRE1 and highMTUS1 (Fig.1g). Of interest, combining MAPRE1 and MTUS1 gene expression allowed better tumor classification compared to each biomarker alone (Fig.1g, Table 1). Together these studies point to MAPRE1 and MTUS1 as valuable diagnostic biomarkers and suggest that analyzing combinatorial expression of these genes may refine tumor classification and improve breast cancer diagnosis.

\section{Combinatorial expression of MAPRE1 and MTUS1 genes correlates with tumor grade}

Analysis of MAPRE1 gene levels in invasive breast carcinomas from 150 patients of the Curie $\underline{\text { Institute (Exploratory Cohort\#2) revealed that MAPRE1 expression is significantly increased }}$ in high grade (GIII) tumors compared to those of histological grade I ( $\mathrm{p}=0.0003)$ and grade II $(\mathrm{p}=0.0242)$ (Fig.2a, Table 2). Tumors were classified into clusters expressing low or high levels of EB1 transcripts according to heat-map analysis of MAPRE1 probesets intensities (Fig.2b). Results indicate that the percentage of grade-III tumors is 3 times more elevated among highMAPRE1 (36,8\%) compared to low-MAPRE1 (10,7\%) expressing tumors (Fig.2c, Table 2). Conversely, grade-I tumors were significantly less abundant among high-MAPRE1 $(18,4 \%)$ compared to low-MAPRE1 $(49,1 \%)$ groups (Table 2), indicating that high transcript levels correlate with high histological grade.

Two independent series of 130 and 155 invasive carcinoma from breast cancer patients of the Curie Institute (Cohort\#3) (Supplemental Table S3) and Gustave Roussy Hospital (Cohort \#4) (Supplemental Table S4) were then analyzed. As shown in Supplemental Fig.S4a, MAPRE1 gene levels were higher in cancer samples compared to 11 normal tissues. In both sets of tumors, MAPRE1 levels were increased in grade-III versus grade-I/II invasive tumors (Supplemental Fig.S4a, S4d). Acordingly, the percentage of high-grade tumors was also elevated in highMAPRE1 compared to low-MAPRE1 breast tumors from Cohorts \#3 and \#4 (Supplemental 
Fig.S4b, S4c, S4e, S4f and Supplemental Table S6, S7), therefore confirming that highMAPRE1 correlates with tumor aggressiveness. Of note, MAPRE1 levels were not significantly different among ER-positive and ER-negative breast tumors of Cohort\#2 (Supplemental Fig.S4g) and Cohort\#4 (Supplemental Fig.S4h), although in Cohort\#3, high MAPRE1 levels were significantly associated with ER-negative tumors (Suppl Fig.S4i and Supplemental Table S6). Of note, the percentage of ER-negative tumors included in Cohort \#2 and \#4 is very low $(18,7 \%$ and $6,6 \%$, respectively) compared to that in Cohort\#3 $(54,6 \%)$, which may explain why $\underline{\text { results do not reach significance in these two series of patients. Future studies including new }}$ $\underline{\text { cohorts with larger numbers of ER-negative breast cancer samples are warranted to address that }}$ question.

We then investigated whether MAPRE2 and MAPRE3 genes may also be regulated in invasive breast cancer. Probesets intensities for these two genes were examined in each cohort of invasive breast carcinomas when data were available, and results (Supplemental Fig.S5) revealed that $M A P R E 2$ and $M A P R E 3$ gene levels do not consistently correlate with tumor grade. Together, these studies indicate that increased levels of MAPRE1, but not MAPRE2 or MAPRE3 genes, correlate with high tumor grade in breast cancer.

Previous studies have shown that MTUS1 gene is down-regulated in breast cancer samples compared with normal tissue, and that low-MTUS1 levels correlate with high tumor grade [17, 18]. We thus investigated the impact of combinatorial MAPRE1 and MTUS1 expression on histological grade. Based on heatmap classification of 150 tumors of Cohort\#2 according to MAPRE1 and MTUS1 probesets intensities, four clusters of tumors expressing either low or high levels of each transcript were established (Fig.2d). Comparison with histological grade revealed that cluster 3 - expressing high-MAPRE1 and low-MTUS1 levels - is significantly associated with higher percentage of high-grade tumors $(44.4 \%$ grade-III tumors in cluster 3 compared to $18.3 \%, 2 \%$ and $18.2 \%$ in clusters 1,2 and 4 , respectively) (Fig.2e, Table 2). 
Consistently, $46.1 \%$ of grade-III tumors were from cluster 3 , compared to $18 \%$ and $6.4 \%$ of grade-II and grade-I tumors, respectively (Fig.2f). Interestingly, among high-MAPRE1 expressing tumors that are the most aggressive, those with low-MTUS1 (cluster 3) were predominantly of high grade compared to those with high-MTUS1 (cluster 4) (Fig.2e), indicating that combined expression of MAPRE1 and MTUS1 genes better distinguishes tumor grade than MAPRE1 alone. Similar results were obtained using two independent sets of tumors (Supplemental Tables S6 and S7, Supplemental Fig.S6). Of note, aggressive tumors from cluster 3 represent a substantial proportion of all breast tumors (18\% in Cohort\#2, 31.5\% in Cohort\#3 and 23.9\% in Cohort\#4) (Table 2 and Supplemental Tables S6 and S7).

\section{Combinatorial expression of MAPRE1 and MTUS1 improves breast cancer prognosis}

The prognostic value of MAPRE1 in breast cancer was assessed by comparing probesets intensities with clinical data of patient survival. The probability of overall survival was significantly reduced in patients with breast tumors expressing high MAPRE1 compared with low MAPRE1 levels (HR 2.22; 95\% CI 1.19-4.12; $\mathrm{p}=0.0058$ ) (Fig.3a and Table 3). The KaplanMeier plotter tool available online was also used to interrogate public databases [28]. High MAPRE1 expression correlated with poorer overall survival in 1402 breast cancer patients (Supplemental Fig.S7a) and poorer relapse-free survival in 3115 patients (Supplemental Fig.S7b), further comforting our results that high-MAPRE1 levels are associated with poor prognosis in breast cancer.

To further investigate whether combinatorial expression of MAPRE1 and MTUS1 may have improved prognostic value compared to each single gene, we evaluated the probability of survival among tumor clusters classified according to MAPRE1 and MTUS1 probeset intensities as defined previously (Fig.2d). Results indicate that overall survival and relapse-free survival are significantly reduced $(\mathrm{p}=0.0001)$ for patients of cluster 3 - expressing high-MAPRE1 and 
low-MTUS1 levels - compared to all other clusters (Fig.3b, 3c, Table 2 and Supplemental

Fig.S7c-d, Table S6). Accordingly, the percentage of patients surviving after 5 years was lower for tumors of cluster $3(42 \%)$ compared to those of cluster $1(61 \%)$, cluster $2(85 \%)$ and cluster $4(60 \%)$ (Fig. 3d). Thus, combining MAPRE1 and MTUS1 gene levels improves breast cancer prognosis compared to MAPRE1 or MTUS1 alone. Multivariate analyses including ER, PR, HER2, tumor grade, MAPRE1 and MTUS1 gene levels further revealed that MAPRE1/MTUS1 gene combination (HR 3.50; CI 1.12-10.85; p 0.015) is an independent indicator of overall survival whereas MAPRE1 alone (HR 1.40; CI 0.40-4.89; p 0.299) is not (Table 3). Notably, MTUS1 (HR 2.7; CI 1.34-5.42; p 0.002) is also identified as an independent prognostic factor, in line with previous studies [18].

We then turned to IHC experiments to evaluate whether combined expression of EB1 and ATIP3 at the protein level may also improve breast cancer prognosis. Experimental conditions set up for EB1 staining (Supplemental Fig.S7e) were used to detect EB1 expression in patient biopsies. As expected, EB1 immunostaining was weak in normal breast and was markedly increased in the cytosol of tumor samples (Supplemental Fig.S7f). Tissue microarrays from 212 invasive breast tumors were then analyzed (Supplemental Table S5) and EB1 immunostaining (Fig.3e) was compared with data for patients survival. Results show that the probability of overall survival was reduced in patients with tumors showing strong EB1 staining (HR 3.46; CI 1.065-7.284; $\mathrm{p}=0.037$ ) (Fig.3f, Table 4), therefore validating the EB1 protein as a prognostic biomarker in breast cancer.

To further evaluate the impact of EB1-ATIP3 combination on patient survival, serial sections of the TMA were stained with anti-ATIP antibodies and samples were classified according to strong or weak EB1 and ATIP3 immunostaining (Fig.3g and Supplemental Table S5). Results indicate that the probability of survival is significantly reduced in patients with tumors expressing strong-EB1 and weak-ATIP3 compared to all other groups ( $\mathrm{p}=0.0153)$ (Fig.3h), 
which comforts the data obtained at the mRNA level (Fig.3b). Consistent with previous results on DNA array analyses, this group of aggressive breast tumors represents $30.4 \%$ of all tumor samples (Table 4). Together, these data confirm DNA array results and demonstrate the improved prognostic value of combinatorial EB1/ATIP3 expression in breast cancer. 


\section{Discussion}

This study examined the diagnostic and prognostic value of MAPRE genes expression in breast cancer, either alone or in combination with candidate tumor suppressor MTUS1 gene. We found that MAPRE1, but not MAPRE2 nor MAPRE3, is up-regulated in malignant tumors compared to benign lesions, and in high-grade compared to low-grade invasive carcinoma. These results are consistent with previous studies [10] reporting EB1 as an oncogenic protein up-regulated in malignant and high-grade breast tumors. In contrast to this latter study however, our data did not clearly correlate EB1 levels with ER status in breast tumors and more studies are required to investigate this question. Results presented here further indicate that high expression of MAPRE1 gene and encoded protein EB1 correlates with reduced survival of the patients, pointing out this molecule as a novel prognostic biomarker in breast cancer.

We further demonstrate here that combinatorial expression of MAPRE1 and MTUS1 genes significantly improves breast cancer diagnosis and prognosis compared to MAPRE1 and MTUS1 alone. Combining data on MAPRE1 and MTUS1 expression both at the gene and protein levels allowed to identify a group of aggressive high-grade tumors with high-EB1 and low-ATIP3 levels, that represent $20 \%$ to $30 \%$ of all breast tumors and are associated with reduced patient survival rates. While a number of studies have identified gene signatures associated with poor prognosis of breast cancer patients [29, 30], this is to our knowledge the first demonstration that combinatorial expression of two defined biomarkers with known associated molecular mechanisms may be used as a tool to select populations of cancer patients for personalized therapy. Further studies are warranted to evaluate the feasibility of using MAPRE1 and MTUS1 biomarkers as diagnostic and prognostic tools in the clinic.

On a molecular basis, these results are supported by our previous findings that ATIP3 acts as a brake on EB1 functions through binding and reducing EB1 turnover at MT growing ends [19]. In the absence of ATIP3, EB1 accumulates at MT ends and forms large comet-like structures 
that specify increased MT dynamics [18]. Results on breast cancer patients favor a model in which in breast tumors with low ATIP3 levels, the brake is turned off and the oncogenic activity of EB1 is increased. This opens the way to novel molecular therapeutic strategies involving delivery of active domains of ATIP3 to target the population of high-EB1/low-ATIP3 breast tumors that remain of poor prognosis. Alternative approaches may rely on the design or discovery of small molecule modulators of EB1 binding at MT ends to compensate for ATIP3 loss in the target population of breast tumors identified here. An integrative approach based on the discovery of small molecule scaffolds that target EB1 interactions with other partners at MT ends has indeed been recently described [31].

In conclusion, we show here for the first time that combinatorial expression of MT-associated EB1 and ATIP3 biomarkers improves breast cancer prognosis compared to each biomarker alone, and may be useful for better stratifying the patients for targeted molecular therapy. Further studies are warranted to analyze combinations of MAPRE1 and MTUS1 genes expression in other types of cancers such as glioblastoma [11], hepatocarcinoma [12], and oral [13] and colorectal [15] cancers, in which high-EB1 protein levels have been shown to correlate with poor clinical outcome.

\section{Acknowledgements}

We wish to thank Dr Céline Lefebvre for helpful discussion and Nicolas Signolle and Ibrahim Bouakka for excellent assistance. This work was supported by the INSERM, the CNRS, the University Paris-Saclay, the Institut Gustave Roussy, the A*MIDEX project ( ${ }^{\circ}$ ANR-11IDEX-0001-02) funded by the "Investissements d'Avenir" French Government program, managed by the ANR and ITMO Cancer AVIESAN as part of the Cancer Plan No. PC201419, the Labex LERMIT, the Fondation ARC pour la Recherche contre le Cancer, the Ligue Nationale Contre le Cancer, the association Odyssea, GEFLUC and Prolific. 


\section{Compliance with ethical standards}

\section{Funding}

This study was funded by the A*MIDEX project ( ${ }^{\circ}$ ANR-11-IDEX-0001-02) funded by the "Investissements d'Avenir" French Government program, managed by the ANR and ITMO Cancer AVIESAN as part of the Cancer Plan No. PC201419. Anne Nehlig was recipient of grants from la Ligue Nationale contre le Cancer, the association Odyssea and Prolific. This study was funded by the Fondation ARC pour la Recherche contre le Cancer, the Labex LERMIT, the association GEFLUC and Prolific.

\section{Compliance with current laws}

The authors declare that all experiments presented here comply with the current laws of France

\section{Conflict of interests :}

The authors declare that they have no conflict of interests.

\section{Ethical approval :}

This article does not contain any studies with animals performed by any of the authors.

All procedures used in studies involving human participants have been previously published elsewhere and were in accordance with ethical standards of the Institut Curie and Gustave Roussy committees, and with 1964 Helsinki declaration.

Informed consent was obtained from all individual participants included in ths study. 


\section{References}

1. Mitchison T, Kirschner M (1984) Dynamic instability of microtubule growth. Nature 312:237-242

2. Howard J, Hyman AA (2003) Dynamics and mechanics of the microtubule plus end. Nature 422:753-758

3. Akhmanova A, Steinmetz MO (2008) Tracking the ends: a dynamic protein network controls the fate of microtubule tips. Nat Rev Mol Cell Biol 9:309-322

4. Su L-K, Qi Y (2001) Characterization of human MAPRE genes and their proteins. Genomics $71: 142-149$

5. Nehlig A, Molina A, Rodrigues-Ferreira S, Honoré S, Nahmias C (2017) Regulation of end-binding protein EB1 in the control of microtubule dynamics. Cell Mol Life Sci 74(13):2381-2393

6. Vaughan KT (2005) TIP maker and TIP marker; EB1 as a master controller of microtubule plus ends. J Cell Biol 171(2) :197-200

7. Maurer SP, Cade NI, Bohner G, Gustafsson N, Boutant E, Surrey T (2014) EB1 accelerates two confirmational transitions important for microtubule maturation and dynamics. Curr Biol 24(4):372-384

8. Galjart N (2010) Plus-end-tracking proteins and their interactions at microtubule ends. Curr Biol 20:R528-R537

9. Jiang K, Toedt G, Montenegro Gouveia S, Davey NE, Hua S, van der Vaart B et al (2012) A proteome- wide screen for Mammalian SxIP Motif-containing microtubule plus-end tracking proteins. Curr Biol 22:1800-1807

10. Kumar P, Wittmann T (2012) +TIPs: SxIPping along microtubule ends. Trends Cell Biol 22:418-428

11. Dong X, Liu F, Sun L, Liu M, Li D, Su D et al (2010) Oncogenic function of microtubule end-binding protein 1 in breast cancer. J Pathol 220(3):361-369

12. Berges R, Baeza-Kallee N, Tabouret E, Chinot O, Petit M, Kruczynski A et al (2014) End-binding 1 protein overexpression correlates with glioblastoma progression and sensitizes to Vinca-alkaloids in vitro and in vivo. Oncotarget 5(24):12769-12787

13. Orimo T, Ojima H, Hiraoka N, Saito S, Kosuge T, Kakisaka T et al (2008) Proteomic profiling reveals the prognostic value of adenomatous polyposis coli-end-binding protein 1 in hepatocellular carcinoma. Hepatology 48(6):1851-1863

14. Kumar M, Mehra S, Thakar A, Shukla NK, Roychoudhary A, Sharma MC et al (2016) End Binding 1 (EB1) overexpression in oral lesions and cancer : A biomarker of tumor progression and poor prognosis. Clin Chim Acta 459:45-52

15. Stypula-Cyrus Y, Mutyal NN, Dela Cruz M, Kunte DP, Radosevich AJ, Wali R et al (2009) End-binding protein 1 (EB1) up-regulation is an early event in colorectal carcinogenesis. FEBS Lett 588(5):829-835

16. Sugihara Y, Taniguchi H, Kushima R, Tsuda H, Kubota D, Ichikawa H et al (2012) Proteomic-based identification of the APC-binding protein EB1 as a candidate of novel tissue biomarker and therapeutic target for colorectal cancer. J Proteomics 75(17) :5342-5355

17. Rodrigues-Ferreira S, Di Tommaso A, Dimitrov A, Cazaubon S, Gruel N, Colasson H et al (2009) 8p22 MTUS1 gene product ATIP3 is a novel anti-mitotic protein underexpressed in invasive breast carcinoma of poor prognosis. PLoS One 4(10): e7239

18. Molina A, Velot L, Ghouinem L, Abdelkarim M, Bouchet BP, Luissint AC et al (2013) ATIP3, a novel prognostic marker of breast cancer patient survival, limits 
cancer cell migration and slows metastatic progression by regulating microtubule dynamics. Cancer Res 73(9):2905-2915

19. Velot L, Molina A, Rodrigues-Ferreira S, Nehlig A, Bouchet BP, Morel M et al (2015) Negative regulation of EB1 turnover at microtubule plus ends by interaction with microtubule-associated protein ATIP3. Oncotarget 6(41):43557-43570

20. André F, Michiels S, Dessen P, Scott V, Suciu V, Uzan C et al (2009) Exonic expression profiling of breast cancer and benign lesions: a retrospective analysis. Lancet Oncol 10(4):381-390

21. Fehlbaum P, Guihal C, Bracco L, Cochet O (2005) A microarray configuration to quantify expression levels and relative abundance of splice variants. Nucleic Acids Res 33:e47

22. Reyal F, Stransky N, Bernard-Pierrot I, Vincent-Salomon A, de Rycke Y, Elvin P et al (2005) Visualizing chromosomes as transcriptome correlation maps: evidence of chromosomal domains containing co-expressed genes-a study of 130 invasive ductal breast carcinomas. Cancer Res 65:1376-1383

23. Hammond ME, Hayes DF, Wolff AC, Mangu PB, Temin S (2010) American society of clinical oncology/college of american pathologists guideline recommendations for immunohistochemical testing of estrogen and progesterone receptors in breast cancer. J Oncol Pract 6(4):195-7

24. Wolff AC, Hammond MEH, Allison KH, Harvey BE, Mangu PB, Bartlett JMS et al. (2018) Human Epidermal Growth Factor Receptor 2 Testing in Breast Cancer: American Society of Clinical Oncology/College of American Pathologists Clinical Practice Guideline Focused Update. J Clin Oncol 36(20):2105-2122

25. Maire V, Némati F, Richardson M, Vincent-Salomon A, Tesson B, Rigaill G et al (2013) Polo-like kinase 1: a potential therapeutic option in combination with conventional chemotherapy for the management of patients with triple-negative breast cancer. Cancer Res 73:816-823

26. Maire V, Baldeyron C, Richardson M, Tesson B, Vincent-Salomon A, Gravier E et al (2013) TTK/hMPS1 is an attractive therapeutic target for triple-negative breast cancer. PloS One 8:e63712

27. Lodillinsky C, Infante E, Guichard A, Chaligné R, Fuhrmann L, Cyrta J et al (2016) p63/MT1-MMP axis is required for in situ to invasive transition in basal-like breast cancer. Oncogene 35(3):344-357

28. Gyorffy B, Lanczky A, Eklund AC, Denkert C, Budczies J, Li Q et al (2010) An online survival analysis tool to rapidly assess the effect of 22,277 genes on breast cancer prognosis using microarray data of 1809 patients. Breast Cancer Res Treat 123:725-731

29. Alexe G, Alexe S, Axelrod DE, Bonates TO, Lozina II, Reiss M et al (2006) Breast cancer prognosis by combinatorial analysis od gene expression data. Breast Cancer Res 8(4):R41

30. Espinosa E, Vara JÁ, Navarro IS, Gámez-Pozo A, Pinto A, Zamora P et al (2011) Gene profiling in breast cancer: time to move forward. Cancer Treat Rev 37(6):416421

31. Almeida TB, Carnell AJ, Barsukov IL, Berry NG (2017) Targeting SxIPEB1 interaction: An integrated approach to the discovery of small molecule modulators of dynamic binding sites. Sci Rep 7(1):15533 


\section{Legends to Figures}

\section{Figure 1: Combinatorial expression of MAPRE1 and MTUS1 genes improves breast cancer diagnosis}

a- Scattered dot plot of MAPRE1 probeset (22919.8.1-T_at) intensity in tumors from patients of cohort \#1 (Exonhit) according to tumor malignancy. $* * \mathrm{p}=0.0016$.

b- Heat-map and hierarchical clustering of 165 breast tumor samples based on the intensities of 6 MAPRE1 probesets (22919.1.1-T_at, 22919.1.2-T_at, 22919.2.1-T_at, 22919.6.1-T_at, 22919.7.1-T_at, 22919.8.1-T_at). Heat-map illustrates relative expression profiles of MAPREl (column) for each tumor sample (line) in continuous color scale from low (blue) to high (red) expression. Dendogram of the 2 selected tumor groups and the corresponding scattered dot plot of MAPRE1 expression are shown on the right.

c- Proportion of patients with benign or malignant tumors according to MAPRE1 level.

d- Scattered dot plot of MTUS1 probeset (57509.5.1-T_at) intensity in tumors from patients of cohort \#1 (Exonhit) according to tumor malignancy. $* * * * p<0.0001$.

e- Heat-map and hierarchical clustering of 165 breast tumor samples based on the intensities of 6 MTUS1 probesets (57509.5.1-T_at, 57509.6.1-T_at, 57509.10.1-T_at, 57509.13.1-T_at, 57509.13.2-T_at, 57509.26.1-T_at). Dendogram of the 2 selected tumor groups and the corresponding scattered dot plot of MTUS1 expression are shown on the right.

f- Proportion of patients with benign or malignant tumors according to MTUS1 level.

g- Proportion of patients with benign or malignant tumors according to combinatorial expression of MAPRE1 and MTUS1 genes.

a-g- Number of samples in each group is indicated under brackets.

\section{Figure 2: Combinatorial expression of MAPRE1 and MTUS1 genes correlates with tumor grade}

a- Scattered dot plot of MAPRE1 probeset (200712_s_at) intensity in tumors from patients of exploratory Cohort \#2 according to tumor grade (GI, GII, GIII). Median value is indicated by the red line. ${ }^{*} \mathrm{p}<0.05, * * * \mathrm{p}<0.001$.

b- Heat-map and hierarchical clustering of 150 breast tumor samples based on the intensities of 2 MAPRE1 probesets (200712_s_at, 200713_s_at). Dendogram of the 2 selected tumor groups and the corresponding scattered dot plot of MAPRE1 expression are shown on the right. 
c- Proportion of patients with Grade I (GI), Grade II (GII) or Grade III (GIII) tumors according to MAPRE1 level.

d- Scattered dot plot of MAPRE1 probeset (200712_s_at, upper panel) and MTUS1 probeset (212093_s_at, lower panel) intensities in tumors classified in 4 clusters of either low or high level of MAPRE1 and MTUS1 transcripts.

e- Proportion of patients with Grade I (GI), Grade II (GII) or Grade III (GIII) tumors according to combinatorial expression of MAPRE1 and MTUS1 in the four clusters.

f- Proportion of patients in clusters 1 to 4 according to tumor grade (GI, GII, GIII).

a-f- Number of samples is indicated under brackets.

\section{Figure 3: Combinatorial expression of MAPRE1 and MTUS1 genes improves breast cancer prognosis}

a- Overall survival curves for patients from exploratory Cohort \#2, with tumors expressing low or high MAPRE1 levels, relative to the dendogram in Fig2b.

b- Overall survival curves for patients from exploratory Cohort \#2, with tumors expressing low or high MAPRE1 and MTUS1 levels, according to clusters 1 to 4 defined in Fig.2d.

c- Relapse-free survival curves for patients from exploratory Cohort \#2, with tumors expressing low or high MAPRE1 and MTUS1 levels as in (b).

d- Proportion of patients remaining alive after 5 years with tumors expressing low or high MAPRE1 and MTUS1 levels as in (b).

e- Representative images of EB1 staining on breast tumor Tissue MicroArray (TMA) using anti-EB1 monoclonal antibody. Numbers on the right upper corner of each image indicate intensity of the EB1 staining from weak $(<1-1)$ to strong EB1 expression $(2-3)$. A bar represents $50 \mu \mathrm{m}$.

f- Overall survival curves for patients from TMA with tumors expressing weak or strong EB1 levels, relative to IHC classification.

g- Representative images of IHC on breast tumor Tissue MicroArray (TMA) using antiEB1 (left) or anti-ATIP3 (right) antibodies showing weak and strong immunostaining. A bar represents $100 \mu \mathrm{m}$.

h- Overall survival curves for patients from TMA, with tumors expressing weak or strong EB1 and ATIP3 levels according to IHC classification.

a-h- Number of tumors in each group is indicated under brackets. 


\section{Legends to Tables}

Table 1. Characteristics of patients from Cohort\#1, with high or low MAPRE1, MTUS1 or combinatorial genes expression

Low and high MAPRE1 and MTUS1 levels were determined according to heatmap analysis of Exonhit probesets intensities. Tumors of Cluster 3 expressing high-MAPRE1 and lowMTUS1 were compared to tumors of all other clusters.

Table 2. Characteristics of patients from Cohort \#2, with high or low MAPRE1 or MAPRE1/MTUS1 combinatorial genes expression

Low and high MAPRE1 levels were determined according to heatmap analysis of Affymetrix probesets intensities. Tumors of Cluster 3 expressing high-MAPRE1 and low-MTUS1 were compared to tumors of all other clusters.

Table 3. Univariate and multivariate analysis of the correlation between clinical parameters, MAPRE1 and MTUS1 levels, and survival time of patients from Cohort\#2

A star indicates multivariate analysis including MAPRE1/MTUS1 combination. HR: Hazard Ratio; CI: Confidence Interval; p: p-value; p-values that reach significance are in bold.

Table 4. Characteristics of patients included in Tissue Microarray analysis, with strong or weak expression of EB1 or EB1/ATIP combinations.

Weak and strong EB1 and ATIP3 levels were scored by IHC as indicated in the Methods. Tumors classified as strong-EB1 and weak-ATIP3 were compared to tumors of all other groups. 
a

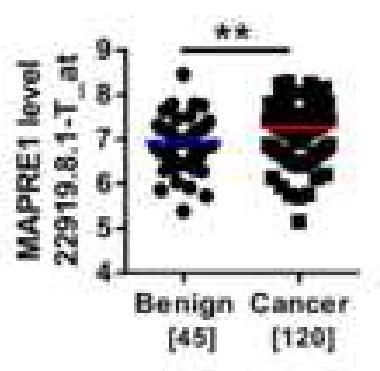

d

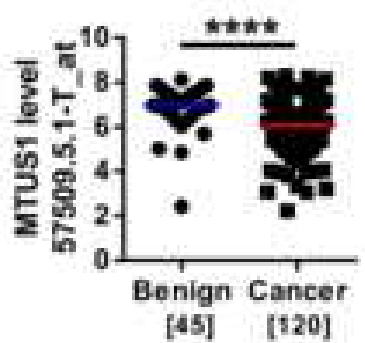

b

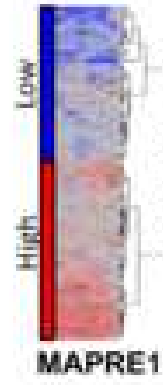

e

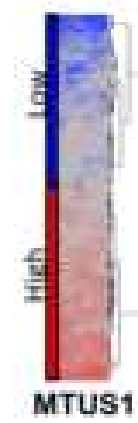

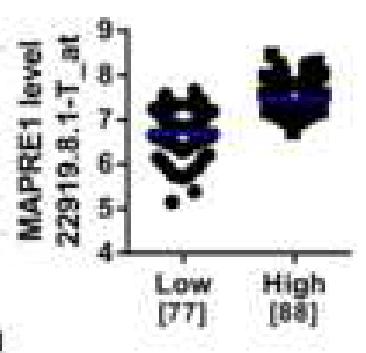

B8

c

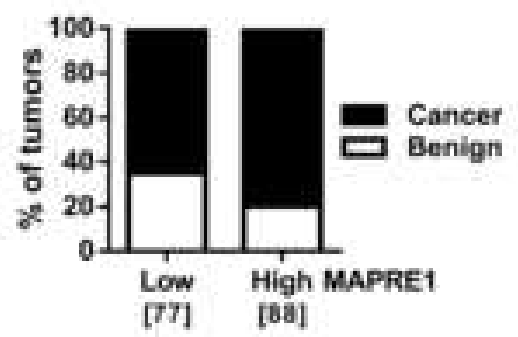

f

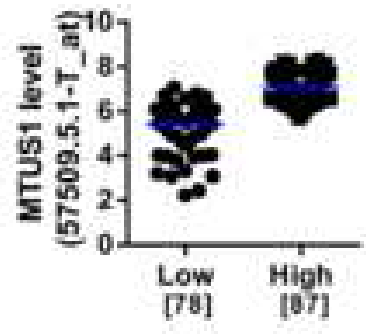

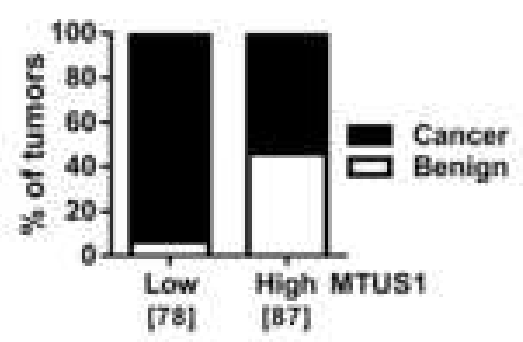

g

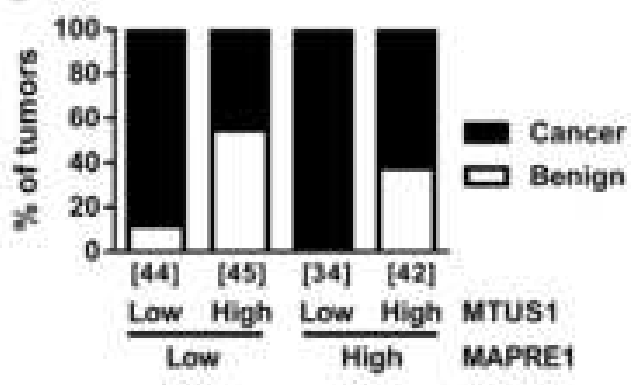

Rodrigues-Ferreira et al. Figure 1 


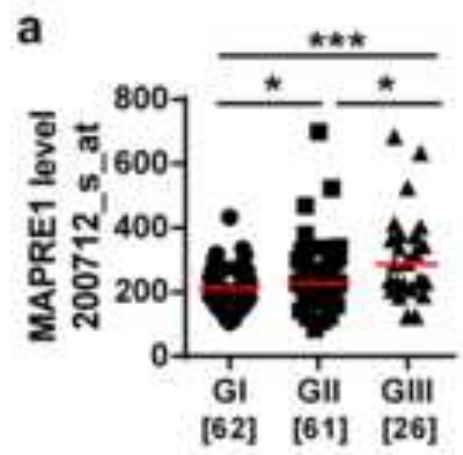

b

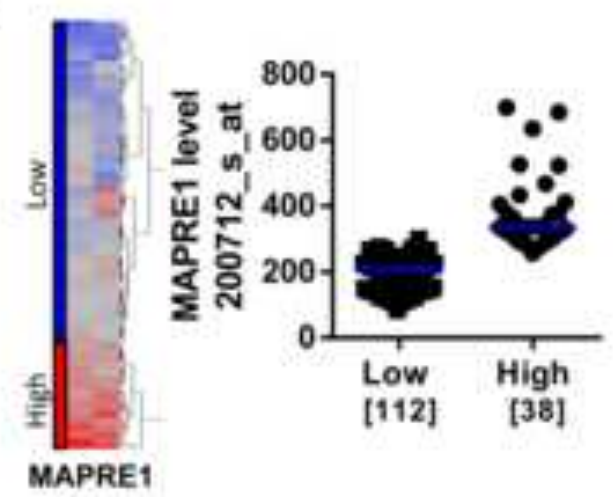

C

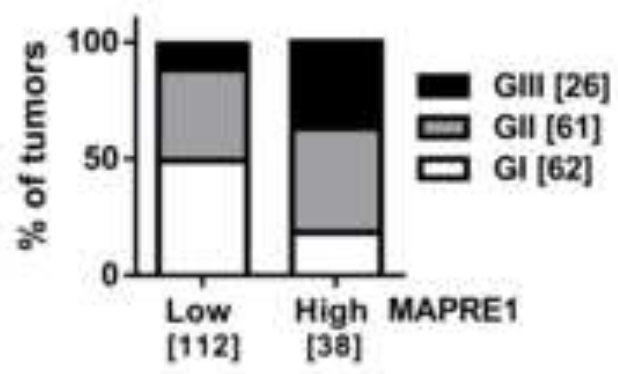

f
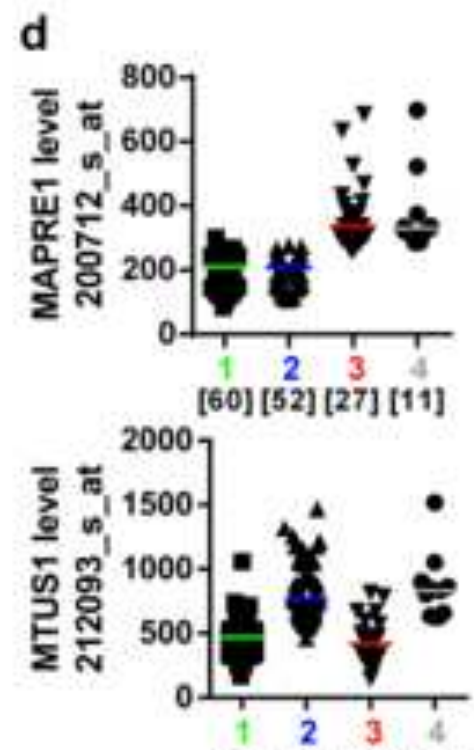

[60][52][27] [11] e

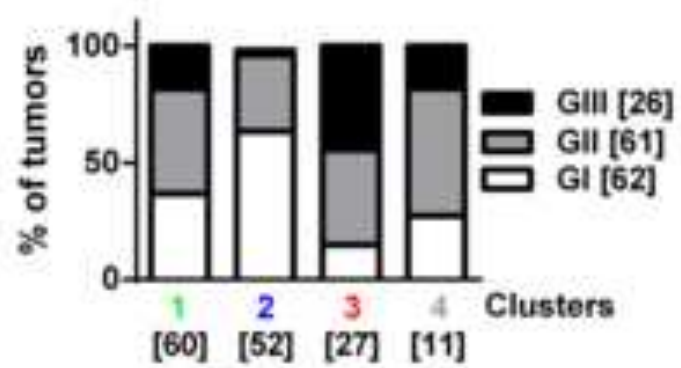

Low High Low High MTUS1

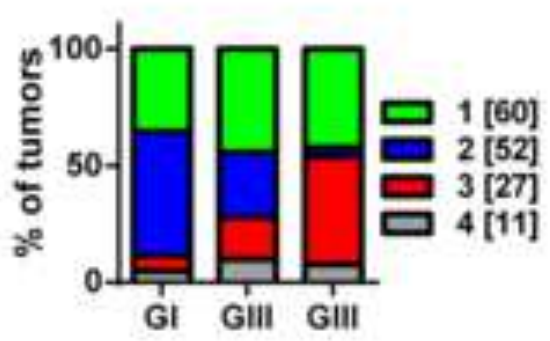
Low High MAPRE1 
a

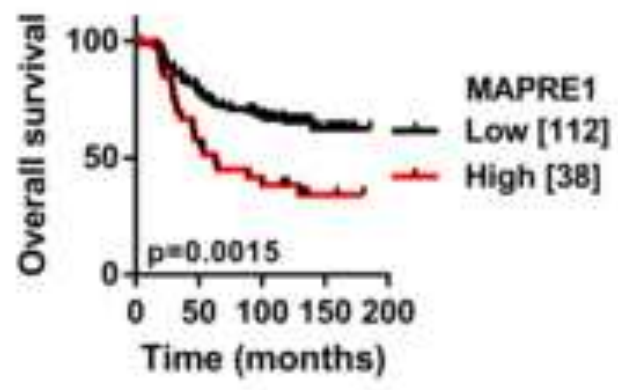

C

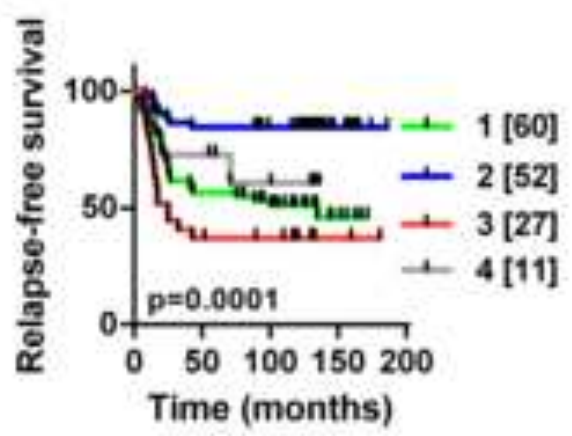

e

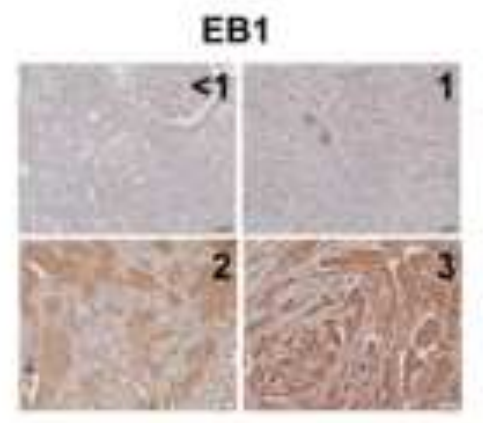

g

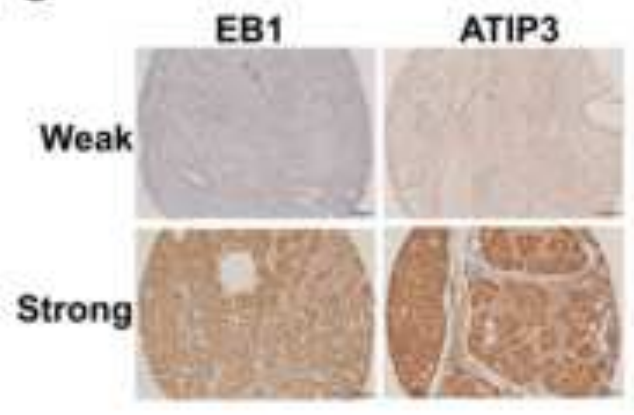

b

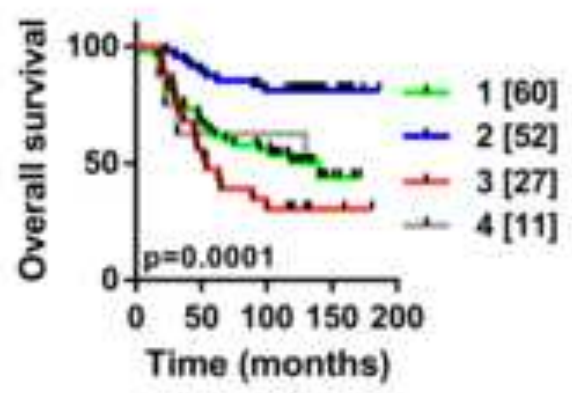

d

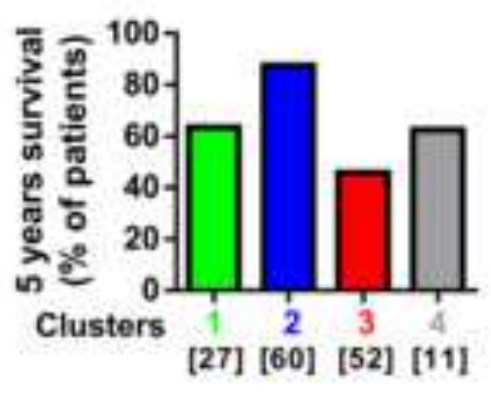

f

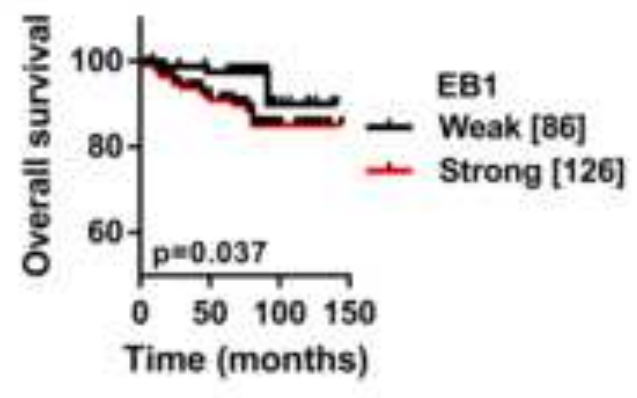

h

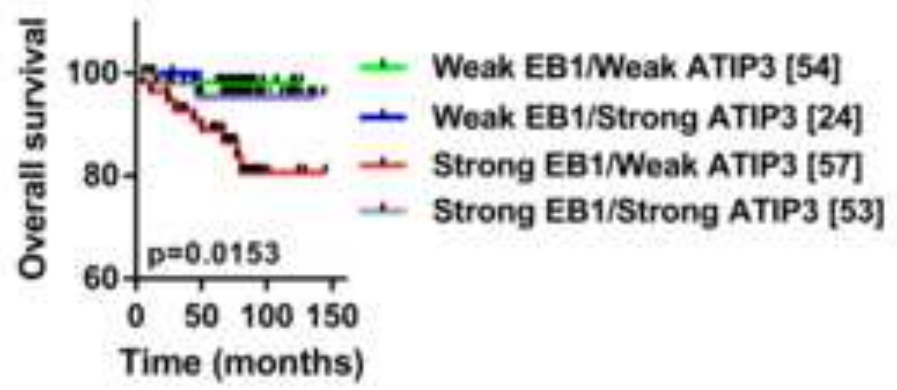




\begin{tabular}{|c|c|c|c|c|c|c|c|c|c|c|}
\hline \multirow[b]{2}{*}{ Variables } & \multirow[b]{2}{*}{$\begin{array}{c}\text { All } \\
\mathrm{N}=165\end{array}$} & \multicolumn{3}{|c|}{ MAPRE1 } & \multicolumn{3}{|c|}{ MTUS1 } & \multicolumn{3}{|c|}{ Combined MAPRE1/MTUS1 } \\
\hline & & $\begin{array}{c}\text { Low } \\
N=77(46,7 \%)\end{array}$ & $\begin{array}{c}\text { High } \\
\mathrm{N}=88(53,3 \%)\end{array}$ & $p$ value & $\begin{array}{c}\text { Low } \\
\mathrm{N}=\mathbf{7 8}(47,3 \%)\end{array}$ & $\begin{array}{c}\text { High } \\
\mathrm{N}=87(52,7 \%)\end{array}$ & $p$ value & $\begin{array}{c}\text { cluster } 3 \\
N=43(26,1 \%)\end{array}$ & $\begin{array}{l}\text { other clusters } \\
\mathrm{N}=122(73,9 \%)\end{array}$ & $\mathrm{p}$ value \\
\hline Benign & $45(27,3 \%)$ & $27(35,1 \%)$ & $18(20,4 \%)$ & \multirow{2}{*}{0,0355} & $5(6,4 \%)$ & $40(46 \%)$ & \multirow{2}{*}{$<0,0001$} & $1(2,3 \%)$ & $44(36,1 \%)$ & \multirow{2}{*}{$<0,0001$} \\
\hline Cancer & $120(72,7 \%)$ & $50(64,9 \%)$ & $70(79,6 \%)$ & & $73(93,6 \%)$ & $47(54 \%)$ & & $42(97,7 \%)$ & $78(63,9 \%$ & \\
\hline
\end{tabular}

Table 1. Characteristics of patients from Cohort\#1 with high or low MAPRE1 , MTUS1 or combined genes expression

Low and high MAPRE1 and MTUS1 levels were determined according to heatmap analysis of probesets intensities.

Tumors of Cluster 3 expressing high-MAPRE1 and low-MTUS1 were compared to tumors of all other clusters 


\begin{tabular}{|c|c|c|c|c|c|c|c|c|}
\hline \multirow{2}{*}{\multicolumn{2}{|c|}{ Variables }} & & \multicolumn{3}{|c|}{ MAPRE1 } & \multicolumn{3}{|c|}{ Combined MAPRE1/MTUS1 } \\
\hline & & $\begin{array}{c}\text { All } \\
N=150\end{array}$ & $\begin{array}{c}\text { Low } \\
\mathrm{N}=112(74,7 \%)\end{array}$ & $\begin{array}{c}\text { High } \\
\mathrm{N}=38(25,3 \%)\end{array}$ & $p$ value & $\begin{array}{c}\text { cluster } 3 \\
N=27(18 \%)\end{array}$ & $\begin{array}{l}\text { other clusters } \\
\mathrm{N}=123(82 \%)\end{array}$ & $p$ value \\
\hline \multirow{3}{*}{ ER } & pos & $119(79,3 \%)$ & $93(83 \%)$ & $26(68,4 \%)$ & \multirow{3}{*}{0,153} & $19(70,4 \%)$ & $100(81,3 \%)$ & \multirow{3}{*}{0,419} \\
\hline & neg & $28(18,7 \%)$ & $17(15,2 \%)$ & $11(28,9 \%)$ & & $7(25,9 \%)$ & $21(17,1 \%)$ & \\
\hline & missing & $3(2 \%)$ & $2(1,8 \%)$ & $1(2,6 \%)$ & & $1(3,7 \%)$ & $2(1,6 \%)$ & \\
\hline \multirow{3}{*}{ PR } & pos & $82(54,7 \%)$ & $67(59,8 \%)$ & $15(39,5 \%)$ & \multirow{3}{*}{0,0712} & $10(37 \%)$ & $72(58,5 \%)$ & \multirow{3}{*}{0,121} \\
\hline & neg & $63(42 \%)$ & $41(36,6 \%)$ & $22(57,9 \%)$ & & $16(59,3 \%)$ & $47(38,2 \%)$ & \\
\hline & missing & $5(3,3 \%)$ & $4(3,6 \%)$ & $1(2,6 \%)$ & & $1(3,7 \%)$ & $4(3,3 \%)$ & \\
\hline \multirow{3}{*}{ HER2 } & pos & $21(14,1 \%)$ & $14(12,5 \%)$ & $7(18,9 \%)$ & \multirow{3}{*}{0,12} & $5(18,5 \%)$ & $16(13,1 \%)$ & \multirow{3}{*}{0,161} \\
\hline & neg & $105(70,5 \%)$ & $77(68,7 \%)$ & $28(75,7 \%)$ & & $21(77,8 \%)$ & $84(68,9 \%)$ & \\
\hline & missing & $23(15,4 \%)$ & $21(18,8 \%)$ & $2(5,4 \%)$ & & $1(3,7 \%)$ & $22(18 \%)$ & \\
\hline \multirow{4}{*}{ Grade } & I & $62(41,3 \%)$ & $55(49,1 \%)$ & $7(18,4 \%)$ & \multirow{4}{*}{0,0004} & $4(14,8 \%)$ & $58(47,2 \%)$ & \multirow{4}{*}{0,0002} \\
\hline & III & $61(40,7 \%)$ & $44(39,3 \%)$ & $17(44,7 \%)$ & & $11(40,7 \%)$ & $50(40,6 \%$ & \\
\hline & III & $26(17,3 \%)$ & $12(10,7 \%)$ & $14(36,8 \%)$ & & $12(44,4 \%)$ & $14(11,4 \%)$ & \\
\hline & missing & $1(0,67 \%)$ & $1(0,67 \%)$ & & & & $1(0,8 \%)$ & \\
\hline \multirow{2}{*}{ recurrence } & no & $91(60,7 \%)$ & $74(66,1 \%)$ & $17(44,7 \%)$ & \multirow{2}{*}{0,02} & $10(37 \%)$ & $81(65,9 \%)$ & \multirow{2}{*}{0,0055} \\
\hline & yes & $59(39,3 \%)$ & $38(33,9 \%)$ & $21(55,3 \%)$ & & 17 (63\%) & $42(34,1 \%)$ & \\
\hline \multirow{3}{*}{ death } & no & $77(51,3 \%)$ & $65(58 \%)$ & $12(31,6 \%)$ & \multirow{3}{*}{0,015} & $8(29,6 \%)$ & $69(56,1 \%)$ & \multirow{3}{*}{0,0097} \\
\hline & \begin{tabular}{|l|} 
yes \\
\end{tabular} & $56(37,3 \%)$ & $35(31,3 \%)$ & $21(55,3 \%)$ & & $17(62,9 \%)$ & $39(31,7 \%)$ & \\
\hline & missing & $17(11,3 \%)$ & $12(10,7 \%)$ & $5(13,2 \%)$ & & $2(7,4 \%)$ & $15(12,2 \%)$ & \\
\hline \multirow{3}{*}{$\begin{array}{l}5 \text {-years } \\
\text { survival }\end{array}$} & no & $41(27,3 \%)$ & $25(22,3 \%)$ & $16(42,1 \%$ & \multirow{3}{*}{0,0228} & $13(48,15 \%)$ & $28(22,8 \%)$ & \multirow{3}{*}{0,0248} \\
\hline & \begin{tabular}{|l} 
yes \\
\end{tabular} & $91(60,7 \%)$ & $75(67 \%)$ & $16(42,1 \%)$ & & $11(40,7 \%)$ & $80(65 \%)$ & \\
\hline & missing & $18(12 \%)$ & $12(10,7 \%)$ & $6(15,8 \%)$ & & $3(11,1 \%)$ & $15(12,2 \%)$ & \\
\hline
\end{tabular}

Table 2. Characteristics of patients from Cohort\#2 with high or low MAPRE1 or combined MAPRE1/MTUS1 genes expression Low and high MAPRE1 and MTUS1 levels were determined according to heatmap analysis of probesets intensities.

Tumors of Cluster 3 expressing high-MAPRE1 and low-MTUS1 were compared to tumors of all other clusters 


\begin{tabular}{|c|c|c|c|c|c|c|c|c|c|}
\hline & \multicolumn{3}{|c|}{ Univariate Analysis } & \multicolumn{3}{|c|}{ Multivariate Analysis } & \multicolumn{3}{|c|}{ Multivariate Analysis* } \\
\hline & HR & $95 \% \mathrm{Cl}$ & $\bar{p}$ & HR & $95 \% \mathrm{Cl}$ & $\mathbf{p}$ & HR & $95 \% \mathrm{Cl}$ & $\mathbf{p}$ \\
\hline ER-vs+ & 3.2 & $1.64-6.2$ & 0.0003 & 1.89 & $0.81-4.36$ & 0.068 & 1.9 & $0.83-4.3$ & 0.062 \\
\hline PR-vs+ & 2.2 & $1.23-3.9$ & 0.0036 & 1.5 & $0.59-3.78$ & 0.195 & 1.5 & $0.59-3.78$ & 0.195 \\
\hline HER2 + vs- & 2.8 & $1.31-5.94$ & 0.0036 & 1.73 & $0.67-4.45$ & 0.128 & 1.67 & $0.61-4.55$ & 0.158 \\
\hline Grade III vs I/II & 4.1 & $1.95-8.6$ & $<0.0001$ & 2,00 & $0.86-4.6$ & 0.051 & 1.98 & $0.86-4.57$ & 0.055 \\
\hline MAPRE1 high vs low & 2.22 & $1.19-4.12$ & 0.0058 & 1.4 & $0.40-4.89$ & 0.299 & & & \\
\hline MTUS1 low vs high & 3.07 & $1.7-5.5$ & 0.0001 & 2.7 & $1.34-5.42$ & 0.0018 & & & \\
\hline MAPRE1 MTUS1 combination cluster 3 vs 2 & 5.46 & $2.12-13.97$ & 0.0002 & & & & 3.5 & $1.12-10.85$ & 0.015 \\
\hline
\end{tabular}

Table 3. Univariate and multivariate analysis of the correlation between clinicopathological parameters, MAPRE1 and MTUS1 level, and survival time of patients from Cohort \#2 * multivariate analysis including MAPRE1/MTUS1 combination

HR: Hazard Ratio, Cl: Confidence Interval, p: p-value

$\mathrm{p}$-values that reach significance are in bold 


\begin{tabular}{|c|c|c|c|c|c|c|c|c|c|}
\hline & & \multicolumn{4}{|c|}{ EB1 } & \multicolumn{4}{|c|}{ Combined EB1/ATIP3 } \\
\hline \multicolumn{2}{|c|}{ Variables } & $\begin{array}{c}\text { All } \\
\mathrm{N}=212\end{array}$ & $\begin{array}{c}\text { Weak } \\
\mathrm{N}=86(40,6 \%)\end{array}$ & $\begin{array}{c}\text { Strong } \\
\mathrm{N}=126(59,4 \%)\end{array}$ & p value & $\begin{array}{c}\text { All } \\
\mathrm{N}=194\end{array}$ & $\begin{array}{c}\text { Strong EB1/Weak } \\
\text { ATIP3 } \\
N=59(30,4 \%)\end{array}$ & \begin{tabular}{|c|} 
other groups \\
$N=135$ \\
$(69,6 \%)$
\end{tabular} & p value \\
\hline ER & $\frac{p o s}{p n e g}$ & $\begin{array}{c}154(72,6 \%) \\
58(27,4 \%)\end{array}$ & $\frac{63(73,3 \%)}{23(26,7 \%)}$ & $\begin{array}{l}91(72,2 \%) \\
35(27,8 \%)\end{array}$ & 0,868 & $\frac{141(72,7 \%)}{53(27,3 \%)}$ & \begin{tabular}{|l|}
$46(78 \%)$ \\
$13(22 \%)$
\end{tabular} & \begin{tabular}{|l|}
$95(70,4 \%)$ \\
$40(29,6 \%)$ \\
\end{tabular} & 0,247 \\
\hline Grade & $\begin{array}{ll}11 \\
11 \\
1\end{array}$ & $\begin{array}{l}38(17,9 \%) \\
76(35,9 \%) \\
\end{array}$ & $\begin{array}{l}19(22,1 \%) \\
3(38,4 \%) \\
32,4\end{array}$ & $\begin{array}{l}19(15,1 \%) \\
4(34,1 \%) \\
\end{array}$ & 0,216 & $\begin{array}{l}32(16,5 \%) \\
73(37,6 \%) \\
\end{array}$ & $\begin{array}{l}11(18,6 \%) \\
21(35,6 \%) \\
2(2, \%)\end{array}$ & \begin{tabular}{|l|}
$21(15,6 \%)$ \\
$52(38,5 \%)$ \\
\end{tabular} & 0,847 \\
\hline \multirow[b]{2}{*}{ death } & $\begin{array}{l}\text { III } \\
\text { no }\end{array}$ & $\frac{98(46,2 \%)}{187(88,2 \%)}$ & $\frac{34(39,5 \%)}{80(93 \%)}$ & $\frac{64(50,8 \%)}{107(84,9 \%)}$ & \multirow{2}{*}{0,043} & $\frac{89(45,9 \%)}{175(90,2 \%)}$ & $\frac{27(45,8 \%)}{48(81,4 \%)}$ & \begin{tabular}{|c|}
$62(45,9 \%)$ \\
$127(94,1 \%)$ \\
\end{tabular} & \multirow[b]{2}{*}{0,0067} \\
\hline & \begin{tabular}{|l|} 
yes \\
missing \\
\end{tabular} & $\begin{array}{l}17(8 \%) \\
8(3,8 \%) \\
\end{array}$ & $\begin{array}{l}3(3,5 \%) \\
3(3,5 \%) \\
\end{array}$ & $\begin{array}{c}14(11,1 \%) \\
5(4 \%) \\
\end{array}$ & & \begin{tabular}{|l}
$13(6,7 \%)$ \\
$6(3,1 \%)$ \\
\end{tabular} & $\begin{array}{l}9(15,3 \%) \\
2(3,4 \%) \\
\end{array}$ & \begin{tabular}{|l|}
$4(3 \%)$ \\
$4(3 \%)$ \\
\end{tabular} & \\
\hline
\end{tabular}

Table 4. Characteristics of patients from TMA, with weak or strong immunostaining of EB1 or combined EB1/ATIP3

Weak and strong EB1 and ATIP3 levels were scored by IHC as indicated in the Methods

Tumors classified as strong-EB1 and weak-ATIP3 were compared to tumors of all other groups. 
Click here to access/download Supplemental Materials - Not to be Published SRF Nahmias supplemental Table S1.xlsx 
Click here to access/download Supplemental Materials - Not to be Published SRF Nahmias supplemental Table S2.xIsx 
Click here to access/download Supplemental Materials - Not to be Published SRF Nahmias supplemental Table S3.xIsx 
Click here to access/download Supplemental Materials - Not to be Published SRF Nahmias supplemental Table S4.xIsx 
Click here to access/download Supplemental Materials - Not to be Published SRF Nahmias supplemental Table S5.xls 
Click here to access/download Supplemental Materials - Not to be Published SRF Nahmias supplemental Table S6.xIsx 
Click here to access/download Supplemental Materials - Not to be Published SRF Nahmias supplemental Table S7.xIsx 
Click here to access/download Supplemental Materials - Not to be Published SRF EB1-ATIP revised supplemental Figures.pdf 
Click here to access/download Supplemental Materials - Not to be Published SRF Nahmias revised Supplemental data BCRT.docx 
Click here to access/download Supplemental Materials - Not to be Published Answer to reviewers comments SRF Nahmias BCRT.docx 